\title{
Radio Halos in galaxy clusters: insight from a mass-selected sample
}

\author{
V. Cuciti* \\ Dipartimento di Fisica e Astronomia, Bologna, Italy \\ INAF-ORA, Bologna, Italy \\ E-mail: vcuciti@ira.inaf.it
}

\begin{abstract}
Giant Radio Halos (RH) are diffuse Mpc-scale synchrotron sources detected in a fraction of massive and merging galaxy clusters. The currently prevailing picture is that RHs trace turbulent regions in the ICM where particles are trapped and accelerated. This scenario predicts a tight connection between the RH properties and the mass and dynamics of the hosting cluster. Large mass-selected samples of galaxy clusters are necessary to explore this connection. We used the Planck SZ cluster catalogue to build up an almost mass-selected sample of galaxy clusters with $M>6 \times 10^{14} M_{\odot}$ and $\mathrm{z}=0.08-0.33$. We looked for the presence of RHs in these clusters in the NVSS at $z<0.2$ and in the Extended GMRT Radio Halo Survey at $z>0.2$. We found evidence of a drop of the fraction of clusters with RHs at lower masses and we confirmed that RHs are generated in merging systems, supporting the idea that mergers play a key role in the formation of RHs. In this respect the next years will be crucial for the understanding of the origin and evolution of RHs, since the new generation of radio telescopes (e.g. LOFAR, MWA, ASKAP) will survey the sky with an unprecedented sensitivity and resolution allowing to test the models in a totally unexplored range of frequencies, RH luminosities, redshift and cluster masses.
\end{abstract}

EXTRA-RADSUR2015 (*)

20-23 October 2015

Bologna, Italy

(*) This conference has been organized with the support of the Ministry of Foreign Affairs and International Cooperation, Directorate General for the Country Promotion (Bilateral Grant Agreement ZA14GR02 - Mapping the Universe on the Pathway to SKA)

\footnotetext{
* Speaker.
} 


\section{Introduction}

Mergers between clusters of galaxies are among the most energetic events in the Universe as they release energies of $\sim 10^{63}-10^{64} \mathrm{erg}$ in a few Gyrs. Part of this energy is channelled into the acceleration of relativistic particles and amplification of magnetic fields in the ICM (see, e.g. [1], for a review). The most spectacular evidence of this activity comes from diffuse Mpc scale synchrotron radio sources, the so-called radio halos $(\mathrm{RH})$. In the current theoretical picture RHs trace turbulent regions in clusters where particles are trapped and re-accelerated during mergers (e.g. [1]). According to this scenario, the formation history of RHs depends on the cluster merging rate throughout cosmic epochs and on the mass of the hosting clusters, which ultimately sets the energy budget available for particle acceleration. In their simplest form, models predict a steepening in the spectra of RHs at a frequency $v_{s}$ that is connected to the energetics of the merger (i.e. on the cluster mass). Therefore, the main expectation is that typical RHs should preferentially be found in massive objects undergoing energetic merging events, whereas they should be rarer in less massive merging-systems and absent in relaxed clusters (e.g. [2] ).

A first statistical measurement of the occurrence of giant RHs in galaxy clusters has been obtained through the "GMRT RH Survey" [3, 4] (hereafter GRHS) and its extension, the EGRHS $[5,6]$. This survey confirmed that RHs are hosted in only $\sim 20-30 \%$ of X-ray luminous $\left(L_{X}(0.1-\right.$ $2.4 \mathrm{keV}) \geq 5 \times 10^{44} \mathrm{erg} / \mathrm{s}$ ) clusters and found that clusters branch into two populations: RHs trace a correlation between $P_{1.4}$ and $L_{X}$, whereas radio-undetected clusters (upper limits) lie about 1 order of magnitude below the correlation (e.g. [7]). Importantly, this bimodal split can be traced to cluster dynamics: RHs are always associated with merging systems, while clusters without RHs are typically relaxed (e.g. [8]).

The recent advent of the Planck cluster surveys, based on the Sunyaev-Zel'dovich (SZ) effect, has enabled the construction of unbiased cluster samples that are almost mass-selected $[9,10]$. The fraction of clusters with RHs appears larger in SZ-selected cluster samples with respect to that derived from X-ray samples [11]. [12] has also demonstrated the presence of a bimodal split in the radio-SZ diagram, for $Y_{500}>6 \times 10^{-5} \mathrm{Mpc}^{2}$. However, the relatively low-mass completeness ( $\sim 50 \%)$ of the [12] sample did not allow the occurrence of RHs to be measured.

Here we give a summary of the statistical radio-X-ray analysis of a large mass-selected sample of galaxy clusters reported in [13].

\section{Cluster sample selection}

In order to provide an unbiased measure of the fraction of clusters hosting RHs and of its dependence on the cluster mass, we used the Planck SZ catalogue (PSZ) [14] to select clusters with $M \gtrsim 6 \times 10^{14} M_{\odot}$ in the redshift range $z \simeq 0.08-0.33$. In this way we obtained a sample of 75 clusters with mass completeness $>80 \%$. Here we report on the statistical analysis of 57 clusters in the sample with available radio observations. The analysis of JVLA and GMRT data of the remaining 18 clusters is in progress. We also report on the analysis of Chandra X-ray data, available for 50 , out of these 57 clusters with the aim to investigate their dynamical status and the connection with the radio properties. 


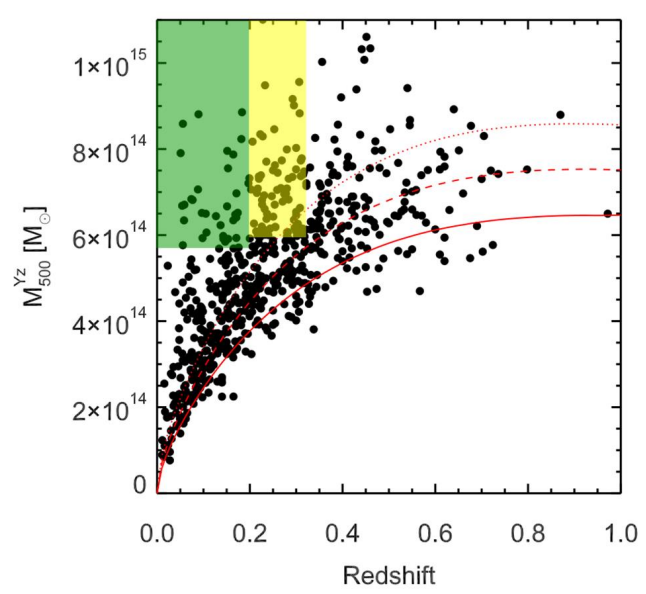

Figure 1: $M_{500}-z$ distribution of the clusters of the PSZ catalogue. The dotted, dashed, and solid lines show the Planck mass limit at 80,50, and $20 \%$ completeness, respectively. The rectangles show the regions where we extracted our sample: green for the low redshift sample, yellow for the high redshift one. Adapted from [14].

Among the 57 clusters, 36 are at $z>0.2$ and belong to the EGRHS, thus have deep radio observations and reliable information about the possible presence of RHs. For the 21 targets in the redshift range $0.08-0.2$ we collected data from the literature (14 clusters) and analysed data from the NVSS radio survey [15] for the remaining seven clusters (see Sect. 3). In Fig.1 we show the $M_{500}-z$ distribution of the Planck clusters: the green and yellow boxes represent the regions where we selected our cluster sample. The low-z sample and the high-z sample are selected from regions with mass-completeness of $\sim 90 \%$ and $80 \%$, respectively. The sample with radio information consists of 57 clusters (listed in [13], Tab. 1) with a completeness in mass of $\sim 63 \%{ }^{1}$.

\section{The low-z sample and the NVSS data analysis}

The low-z sample includes 21 clusters with $0.08<z<0.2$ and $M_{500} \gtrsim 5.7 \times 10^{14} M_{\odot}$. For 14 of these clusters we found information in the literature on the presence or absence of clusterscale radio emission that is based on pointed VLA/WSRT observations. We reprocessed the NVSS fields of the seven clusters without literature information with the aim of improving the quality of the radio images, i.e. lowering the rms noise and reducing the contribution of noise pattern. For the reprocessed clusters we reached an average $\mathrm{rms} \approx 0.25 \mathrm{mJy} / \mathrm{beam}$, which is $\sim 2$ times better than the nominal NVSS noise.

In order to investigate the possible presence of residual emission in the central regions of these clusters we selected on each map a $1 \mathrm{Mpc}$ circle centred on the centroid of the cluster Xray emission, then, with the task BLANK, we masked the discrete sources in the cluster and we measured the residual diffuse flux density (RDF) in the circular region. We compared the RDF with the flux densities measured in other areas of the same size taken around the cluster (three for each cluster), i.e. "control fields" ${ }^{2}$. We measured the offset between the RDF and the average value of

\footnotetext{
${ }^{1}$ This is estimated as $\frac{21+36}{(21 / 0.9)+(54 / 0.8)} \sim 63 \%$

${ }^{2}$ In order to make a consistent comparison we normalized both the RDF and the control field flux densities (CFF) to the number of pixels enclosed in a circle of $1 \mathrm{Mpc}$ diameter after masking the discrete sources
} 
the CFF and we found that four clusters show an excess at $>2 \sigma$ level. We consider this to be the threshold level at which to identify clusters with the possible presence of a RH.

With our procedure, based on the NVSS, we identified four cases with suspect diffuse radio emission, however, we cannot confirm the presence of RHs in these clusters. Follow up GMRT and JVLA observations of these clusters are ongoing.

\section{Occurrence of radio halos}

The aim of this Section is to derive the occurrence of RHs as a function of the mass of the hosting clusters. Among the sample of 57 clusters with radio information, 24 ( 7 at $z<0.2$ and 17 at $z>0.2$ ) host RHs and four low-z clusters show residual emission in a Mpc-scale region that is a possible indication of the presence of a RH (Sect. 3). We split this sample into two mass bins and derived the fraction of clusters with RHs, $f_{R H}$, in the low-mass bin (LM, $M<M_{\text {lim }}$ ) and in the high-mass bin (HM, $M>M_{\text {lim }}$ ) assuming different values of the limiting mass, $M_{\text {lim }}$ (as detailed below). In general we found that $f_{R H}$ is lower in the LM bins $\left(f_{R H} \approx 20-30 \%\right)$, while it is higher $\left(f_{R H} \approx 60-80 \%\right)$ in the HM bins (Fig. 2). This difference is systematic and thus we attempted to identify the value of $M_{\text {lim }}$ that provides the most significant jump between low- and high-mass clusters.

We performed Monte Carlo simulations considering the four objects in the low-z sample with suspect diffuse emission as non-RH clusters (case (i)) and (ii) as RH clusters. Considering case (i), we randomly assigned 24 RHs among the 57 clusters of the sample and obtained the distributions of RHs in the two mass bins (after $10^{5}$ trials), the same distributions expected if the RHs had been distributed independently of the cluster mass. We consider five different values of the transition mass between the two bins, specifically $M_{\text {lim }}=(6,7,8,9,10) \times 10^{14} M_{\odot}$. Fig. 2 shows the observed fraction of RHs (dots) together with the results of the Monte Carlo simulations (shadowed regions) in the HM bin (left panel) and in the LM bin (right panel). We show the measured fraction of cluster
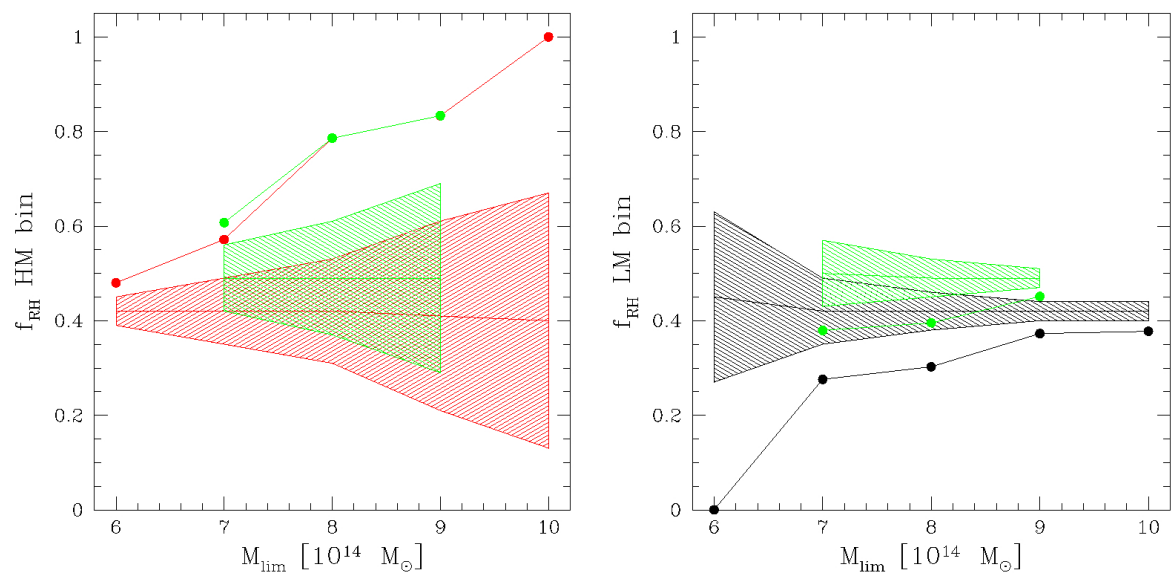

Figure 2: Observed fraction of RHs $f_{R H}$ (dots) compared to the value predicted by the Monte Carlo simulations (shadowed regions) in the HM bin (left panel) and in the LM bin (right panel) as a function of the limiting mass $M_{\text {lim }}$. In both panels the green dots and the green shadowed regions show the case in which the four clusters with suspect diffuse emission are considered as RH clusters. 
with RHs and the results of the Monte Carlo analysis in cases ( $i$ ) (red and black dots and shadowed regions) and (ii) (green dots and shadowed regions). For a clearer visualization in Fig. 2, for case (ii) we only show the results obtained by assuming $M_{\text {lim }}=(7,8,9) \times 10^{14} M_{\odot}$. Fig. 2 shows that in the HM bin the observed $f_{R H}$ is always greater than that predicted by the Monte Carlo simulations; on the contrary, in the LM bin the observed $f_{R H}$ is always lower than that predicted by the Monte Carlo analysis. This suggests the existence of a systematic drop of $f_{R H}$ in low-mass systems.

In both cases $(i)$ and $(i i)$, we found that the value of $M_{\text {lim }}$ that gives the most significant result and maximizes the drop of $f_{R H}$ between the two mass bins is $M_{\text {lim }} \approx 8 \times 10^{14} M_{\odot}$, for which $f_{R H} \simeq 30 \%$ (40\%) in the LM bin and $f_{R H} \simeq 79 \%(79 \%)$ in the HM bin in case (i) (in case (ii)). For $M_{\text {lim }} \approx$ $8 \times 10^{14} M_{\odot}$ the observed $f_{R H}$ in the two mass bins differs from that obtained by the Monte Carlo analysis at $\sim 3.2 \sigma$ in case $(i)$ and $\sim 2.5 \sigma$ in case (ii). This means that the chance probability of the observed drop of $f_{R H}$ is $<7.4 \times 10^{-4}(i)$ and $<5.7 \times 10^{-3}$ (ii).

Based on this analysis we conclude that there is statistical evidence for a drop of the fraction of RHs in galaxy clusters at smaller masses. This is the first time that such an indication is derived using a mass-selected sample.

According to current modellings mergers in smaller systems may not have sufficient energy to generate RHs and a drop of the occurrence of RHs in smaller clusters is expected $[2,16,1]$. In Fig. 3 we compare our results with the theoretical expectations based on [17,2]. Despite the crude approximations adopted in these models, there is an overall agreement between the observed and predicted behaviour of $f_{R H}$ with the cluster mass. The model slightly underestimates $f_{R H}$ in the high-mass bin. There are two main reasons for this: 1) the use of the Press-Schechter formalism [18], which is well known to underestimate the merging rate, and hence the number density, of very massive systems; and 2) the fact that the model predictions do not include RHs with very steep radio spectra, i.e. those with steepening frequency $v_{s} \lesssim 600 \mathrm{MHz}$.

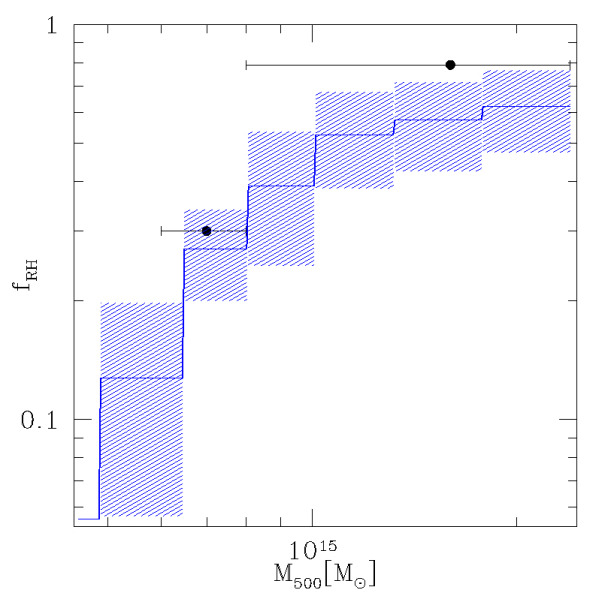

Figure 3: Expected fraction of clusters with RHs with steepening frequency [8] $v_{s}>600 \mathrm{MHz}$ in the redshift range $0.08<z<0.33$ (blue line and shadowed region). Calculations have been performed for the following choice of model parameters: $b=1.5,\langle B\rangle=1.9 \mu \mathrm{G}$ (where $B=\langle B\rangle \times(M /\langle M\rangle)^{b}$ ), and $\eta_{t}=0.2$ (see [19] and referencees therein). The observed fraction of clusters with RHs in the two mass bins is also shown (black points with horizontal error bars). 


\section{Radio halo-cluster merger connection}

In this section we investigate the connection between the presence or absence of RHs in clusters and the cluster dynamical status of merger or relaxed. 50 cluster, out of 57 have available Chandra X-ray data, that we reduced and analysed following $[8,12]$. We studied the cluster substructures on the RH scale analysing the surface brightness inside an aperture radius $R_{a p}=500$ kpc. We used three main indicators of the level of dynamical disturbance in clusters: the power ratios (e.g. [20, 21]), the emission centroid shift (e.g. [20, 22, 21]), and the surface brightness concentration parameter (e.g. [23]).

Fig. 4 shows that RH clusters (red dots) can be separated from clusters without RH (black dots) in the morphological diagrams: RHs are associated with dynamically disturbed clusters, while the vast majority of clusters without Mpc-scale diffuse radio emission are relaxed objects.

About $80 \%$ of the clusters in the HM bin of our sample are mergers, and this explains why RHs are fairly common in this bin (Sect. 4). At least ten merging clusters of our sample do not host RHs. In fact, the generation of turbulence in the ICM as a consequence of merger events takes some time, corresponding to a "switch on", "switch off" phase of RHs. During these phases $\mathrm{X}$-ray and radio properties of clusters can be unconnected, since RHs would be underluminous or absent, whereas the hosting cluster would appear disturbed in the X-rays (e.g. [24]). An additional possibility is that some of the dynamically disturbed systems host RHs with very steep spectra that are not easily seen at our observing frequencies $([25,16])$. Indeed the great majority of merging clusters without RHs belong to the LM bin, which might support the idea that in these cases (or some of them) the energy provided by the merger is not sufficient to generate RHs emitting at the observing frequencies.
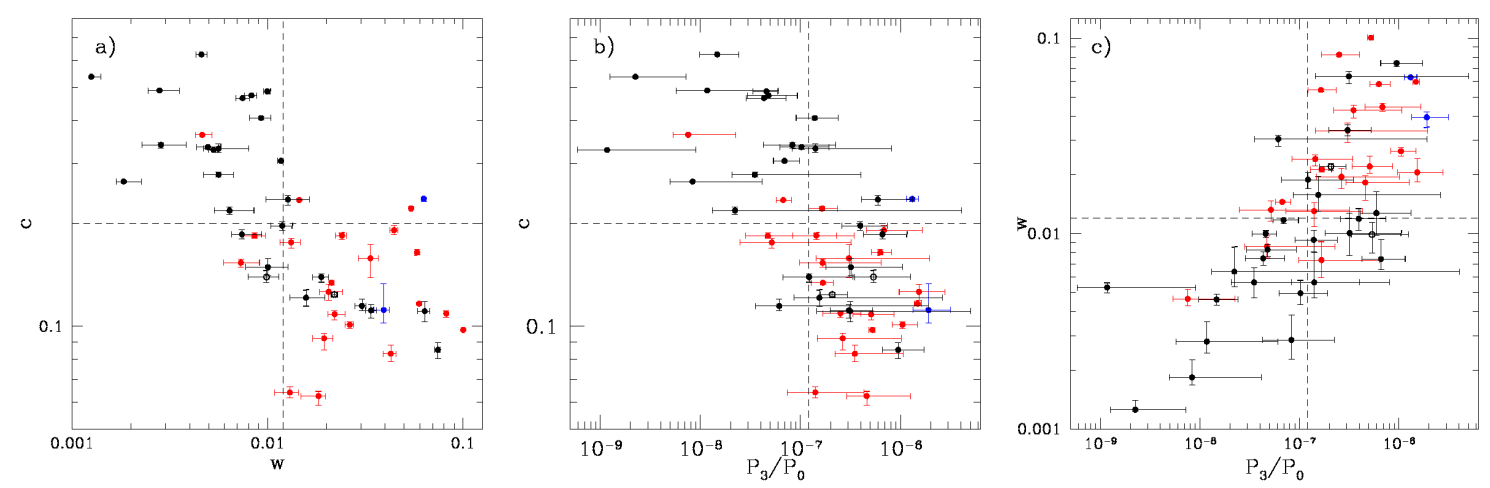

Figure 4: (a) $c-w$, (b) $c-P_{3} / P_{0}$, (c) $w-P_{3} / P_{0}$ diagrams. Vertical and horizontal dashed lines: $c=0.2, w=0.012$, and $P_{3} / P_{0}=1.2 \times 10^{-7}$ [8]. Red, black, and blue dots represent clusters with RH, clusters without RH, and clusters hosting relics (without RHs), respectively. Black open dots are clusters with suspect diffuse emission from the NVSS.

\section{Summary}

We have presented a step toward an unbiased analysis of the occurrence of RHs as a function of the cluster mass in a mass-selected sample of galaxy clusters. We built a sample of 75 clusters with $M \gtrsim 6 \times 10^{14} M_{\odot}$ in the redshift range $0.08<z<0.33$ selected from the Planck SZ catalogue. 
Here we have analysed a subsample of 57 clusters that have available radio information. The completeness in mass of this sample is $\sim 63 \%$.

We split our sample into two mass bins, the low-mass bin (LM, $M<M_{\text {lim }}$ ) and the high-mass bin (HM, $M>M_{\text {lim }}$ ), and derived the fraction of clusters with RHs in the two mass bins for different values of $M_{\text {lim }}$, finding that $f_{R H}$ is $\approx 60-80 \%$ in the HM bin and $\approx 20-30 \%$ in the LM bin. By means of Monte Carlo simulations we obtained the distributions of RHs in the two mass bins (after $10^{5}$ trials), expected in the case that RHs were distributed independently of the cluster mass. We found that for $M_{\text {lim }} \approx 8 \times 10^{14} M_{\odot}$ the observed $f_{R H}$ in the two mass bins differs from that obtained by the Monte Carlo analysis with a significance that ranges between $2.5 \sigma$ and $3.2 \sigma$, which means that the probability of obtaining the observed drop of $f_{R H}$ by chance is $<5.7 \times 10^{-3}$ or even lower (see Sect 4). This suggests that the increase of the occurrence of RHs with the cluster mass is likely to be real, rather than by chance.

The drop of the fraction of clusters hosting RH for less massive systems is naturally and uniquely expected in the framework of turbulent re-acceleration models (e.g. [17]) that provide a popular picture for the formation of giant RHs in galaxy clusters. A solid comparison between models and our observations is still premature owing to the incompleteness of the observed sample. Still, with this caveat in mind, in Fig. 3 we have shown that there is a quite good agreement between models and observations.

We analysed the Chandra X-ray data, available for 50 clusters, and we derived the morphological parameters (the centroid shift, $w$; the power ratio, $P_{3} / P_{0}$; and the concentration parameter, $c$ ), which are powerful diagnostics of the cluster dynamical status. We confirmed that RHs are hosted by merging clusters, while the majority of non-RH clusters are relaxed, thus highlighting the crucial role that merger events play in the origin of RHs.

We are currently analysing GMRT and the JVLA proprietary data of the remaining 18 clusters in the sample in order to achieve a conclusive measure of the occurrence of RHs in a mass-selected sample of galaxy clusters.

\section{References}

[1] G. Brunetti and T. W. Jones, Cosmic Rays in Galaxy Clusters and Their Nonthermal Emission, International Journal of Modern Physics D 23 (Mar., 2014) 1430007-98, [1401. 7519].

[2] R. Cassano, G. Brunetti and G. Setti, Statistics of giant radio haloes from electron reacceleration models, MNRAS 369 (July, 2006) 1577-1595, [astro-ph/ 0604103 ].

[3] T. Venturi, S. Giacintucci, G. Brunetti, R. Cassano et al., GMRT radio halo survey in galaxy clusters at $z=$ 0.2-0.4. I. The REFLEX sub-sample, A\&A 463 (Mar., 2007) 937-947, [astro-ph/ 0610271 ].

[4] T. Venturi, S. Giacintucci, D. Dallacasa, R. Cassano et al., GMRT radio halo survey in galaxy clusters at $z=0.2-0.4$. II. The eBCS clusters and analysis of the complete sample, A\&A 484 (June, 2008) 327-340, [0803.4084].

[5] R. Kale, T. Venturi, S. Giacintucci, D. Dallacasa et al., The Extended GMRT Radio Halo Survey. I. New upper limits on radio halos and mini-halos, A\&A 557 (Sept., 2013) A99, [1306.3102].

[6] R. Kale, T. Venturi, S. Giacintucci, D. Dallacasa et al., The Extended GMRT Radio Halo Survey. II. Further results and analysis of the full sample, A\&A $\mathbf{5 7 9}$ (July, 2015) A92, [1503. 02415]. 
[7] G. Brunetti, T. Venturi, D. Dallacasa, R. Cassano et al., Cosmic Rays and Radio Halos in Galaxy Clusters: New Constraints from Radio Observations, ApJ 670 (Nov., 2007) L5-L8, [0 710.0801$].$

[8] R. Cassano, S. Ettori, S. Giacintucci, G. Brunetti et al., On the Connection Between Giant Radio Halos and Cluster Mergers, ApJ 721 (Oct., 2010) L82-L85, [1008. 3624 ].

[9] P. M. Motl, E. J. Hallman, J. O. Burns and M. L. Norman, The Integrated Sunyaev-Zeldovich Effect as a Superior Method for Measuring the Mass of Clusters of Galaxies, ApJ 623 (Apr., 2005) L63-L66, [astro-ph/0502226].

[10] D. Nagai, The Impact of Galaxy Formation on the Sunyaev-Zel'dovich Effect of Galaxy Clusters, ApJ 650 (Oct., 2006) 538-549, [astro-ph/0512208].

[11] M. W. Sommer and K. Basu, A comparative study of radio halo occurrence in SZ and X-ray selected galaxy cluster samples, MNRAS 437 (Jan., 2014) 2163-2179, [1307 . 304 9].

[12] R. Cassano, S. Ettori, G. Brunetti, S. Giacintucci et al., Revisiting Scaling Relations for Giant Radio Halos in Galaxy Clusters, ApJ 777 (Nov., 2013) 141, [1306.4379].

[13] V. Cuciti, R. Cassano, G. Brunetti, D. Dallacasa et al., Occurrence of radio halos in galaxy clusters. Insight from a mass-selected sample, A\&A $\mathbf{5 8 0}$ (Aug., 2015) A97, [1506.03209].

[14] Planck Collaboration, P. A. R. Ade, M. Arnaud, M. Ashdown et al., Planck 2013 results. XXXI. Consistency of the Planck data, A\&A 571 (Nov., 2014) A31, [1508.03375].

[15] J. J. Condon, W. D. Cotton, E. W. Greisen, Q. F. Yin et al., The NRAO VLA Sky Survey, AJ 115 (May, 1998) 1693-1716.

[16] G. Brunetti, S. Giacintucci, R. Cassano, W. Lane et al., A low-frequency radio halo associated with a cluster of galaxies, Nature 455 (Oct., 2008) 944-947, [0 810 . 4288].

[17] R. Cassano and G. Brunetti, Cluster mergers and non-thermal phenomena: a statistical magneto-turbulent model, MNRAS 357 (Mar., 2005) 1313-1329, [astro-ph/ 0412475 ].

[18] W. H. Press and P. Schechter, Formation of Galaxies and Clusters of Galaxies by Self-Similar Gravitational Condensation, ApJ 187 (Feb., 1974) 425-438.

[19] R. Cassano, G. Brunetti, R. P. Norris, H. J. A. Röttgering et al., Radio halos in future surveys in the radio continuum, A\&A $\mathbf{5 4 8}$ (Dec., 2012) A100, [1210.1020].

[20] D. A. Ventimiglia, G. M. Voit, M. Donahue and S. Ameglio, Substructure and Scatter in the Mass-Temperature Relations of Simulated Clusters, ApJ 685 (Sept., 2008) 118-127, [0806. 0850 ].

[21] H. Böhringer, G. W. Pratt, M. Arnaud, S. Borgani et al., Substructure of the galaxy clusters in the REXCESS sample: observed statistics and comparison to numerical simulations, A\&A 514 (May, 2010) A32, [0912.4667].

[22] B. J. Maughan, L. R. Jones, M. Pierre, S. Andreon et al., Testing the galaxy cluster mass-observable relations at $z=1$ with XMM-Newton and Chandra observations of XLSSJ022403.9-041328, MNRAS 387 (July, 2008) 998-1006, [0 709 .2300].

[23] J. S. Santos, P. Rosati, P. Tozzi, H. Böhringer et al., Searching for cool core clusters at high redshift, A\&A 483 (May, 2008) 35-47, [0 802 . 1445].

[24] J. Donnert, K. Dolag, G. Brunetti and R. Cassano, Rise and fall of radio haloes in simulated merging galaxy clusters, MNRAS 429 (Mar., 2013) 3564-3569, [1211.3337].

[25] R. Cassano, G. Brunetti and G. Setti, Statistics of giant radio haloes from electron reacceleration models, MNRAS 369 (July, 2006) 1577-1595, [astro-ph / 0604103 ]. 